\title{
Riemann-Liouville processes arising from Branching particle systems
}

\author{
Yuqiang LI * \\ School of Finance and Statistics, East China Normal University, \\ Shanghai 200241, P. R. China.
}

\begin{abstract}
It is proved in this paper that Riemann-Liouville processes can arise from the temporal structures of the scaled occupation time fluctuation limits of the sitedependent $(d, \alpha, \sigma(x))$ branching particle systems in the case of $1=d<\alpha<2$ and $\int_{\mathbb{R}} \sigma(x) \mathrm{d} x<\infty$.
\end{abstract}

Keywords: Functional limit theorem; Branching particle system; Riemann-Liouville process

AMS 2000 Subject Classification: 60F17; 60J80

\section{Introduction}

A Riemann-Liouville process (RL process) is a centered Gaussian process. It can be represented by the moving average representation of Brownian motion

$$
\int_{0}^{t}(t-u)^{H-1 / 2} B(\mathrm{~d} u), \quad 0 \leq t<\infty
$$

Here $B(t)$ denotes the standard Brownian motion and $H>0$ is a constant. For convenience, we denote the RL process by $\left\{R^{H}(t), t \geq 0\right\}$. It is easy to see from (1.1) that $R^{H}$ does not have stationary increments but is self-similar with index $H$. RL processes are very useful and important because they are closely related to the Riemann-Liouville derivative of Gaussian noise. As early as 1950s, Lévy [1] had briefly commented on this kind of processes. Since then, much work has been done in order to study it; see, for example, [10, 15, 18, and the references therein.

From (1.1) one can readily think of the fractional Brownian motion (FBM) $B^{H}$ with (Hurst) index $H \in(0,1)$. The FBM $B^{H}$ has a moving average representation as follows.

$$
B^{H}(t):=\int_{-\infty}^{t}\left[(t-u)_{+}^{H-1 / 2}-(-u)_{+}^{H-1 / 2}\right] B(\mathrm{~d} u)
$$

where $u_{+}=u \vee 0$. It is well-known that $B^{H}$ is a centered Gaussian process with the covariance function

$$
\mathbb{E}\left[B^{H}(t) B^{H}(s)\right]=\frac{1}{2}\left(|s|^{2 H}+|t|^{2 H}-|t-s|^{2 H}\right) .
$$

${ }^{*}$ Research is supported partially by NSFC grant (10901054) and ECSC grant (13zz037). 
and $B^{H}$ is self-similar with index $H$ and has stationary increments.

Sub-fractional Brownian motions (Sub-FBMs) were formally introduced in 2004 independently by Bojdecki et al [3] and Dzhaparidze and van Zanten [9], respectively. The Sub-FBM $\left\{\eta^{H}(t), t \geq 0\right\}$ with index $H \in(0,1)$ is also a centered Gaussian process with the covariance function

$$
\mathbb{E}\left[\eta^{H}(t) \eta^{H}(s)\right]=|s|^{2 H}+|t|^{2 H}-\frac{1}{2}\left((s+t)^{2 H}+|t-s|^{2 H}\right) .
$$

This kind of processes has many properties which are the same as those of FBMs, but its increments are not stationary.

When $H \neq 1 / 2$, RL processes, FBMs and Sub-FBMs share the same property that their increments on non-overlapping intervals are correlated. In fact, Bojdecki et al 3 showed that the covariance of increments on intervals at distance $t$ decays like $t^{2 H-3}$ for the Sub-FBM $\eta^{H}$ and $t^{2 H-2}$ for the FBM $B^{H}$. In addition, as we will point out in Proposition 2.1, this index for the RL process $R^{H}$ is $t^{H-3 / 2}$.

Bojdecki et al [3, 4] studied the functional limits of the occupation time fluctuation processes

$$
X_{T}(t)=\frac{1}{F_{T}} \int_{0}^{T t}(N(s)-\mathbb{E}(N(s))) \mathrm{d} s, \quad t \geq 0,
$$

of a classical $(d, \alpha, 1)$-branching particle system, where $N(t)$ denotes its random counting measure at time $t$, and found that under the assumptions $\alpha<d<2 \alpha$ and $F_{T}=T^{\frac{3}{2}-\frac{d}{2 \alpha}}$, a Sub-FBM $\eta^{H}$ with $H=\frac{3}{2}-\frac{d}{2 \alpha}$ can arise from the temporal structure of the limit process of $X_{T}(t)$. Here $\mathbb{E}(N(s))$ is the expectation function understood as $\langle\mathbb{E}(N(s)), \phi\rangle=\mathbb{E}(\langle N(s), \phi\rangle)$ for any $\phi \in \mathcal{S}\left(\mathbb{R}^{d}\right)$, the space of smooth rapidly decreasing functions. Motivated by the aforementioned works, the main purpose of this paper is to report a similar but new result. We show that under certain conditions, RL processes arise from the limits of occupation time fluctuations of the so-called site-dependent branching particle systems (see $\mathrm{Li}$ [14] for details). This result is not obvious at a first sight. In fact, so far as we know, it is not formally reported in existing literature even if RL processes have been explored for longer time than that of FBMs and Sub-FBMs.

Precisely, in this paper, we consider a branching particle system in $\mathbb{R}$, whose particles start off at time $t=0$ from a Poisson random field with Lebesgue intensity measure $\lambda$, evolve independently with a symmetric $\alpha$-stable Lévy motion and undergo at rate $\gamma$ the critical, finite variance branching which depends on the position $x$ where the particle splits and is controlled by the moment generating function

$$
g(s, x)=s+\sigma(x)(1-s)^{2}, \quad 0 \leq s \leq 1,0 \leq \sigma(x) \leq 1 / 2, x \in \mathbb{R},
$$

where $\sigma(\cdot)$ is a measurable function on $\mathbb{R}$. For convenience, we refer this model to the $(1, \alpha, \sigma(x))$-particle system. Let $N(s)$ denote the random counting measure of the $(1, \alpha, \sigma(x))$-particle system. Consider the scaled occupation time fluctuation process defined by (1.5). We prove that when $\alpha \in(1,2)$, and $F_{T}=T^{\frac{3}{2}-\frac{1}{\alpha}}$, the RL process $R^{H}$ with $H=\frac{3}{2}-\frac{1}{\alpha}$ arises from the temporal structure of the fluctuation limit process if $\int_{\mathbb{R}} \sigma(x) \mathrm{d} x<\infty$. In addition, we also prove that the standard Brownian motion can arise from the limit process in the case of $\alpha=1$ if $F_{T}=T^{1 / 2} \ln T$ and $\int_{\mathbb{R}} \sigma(x) \mathrm{d} x<\infty$. 
There is much other literature in the field of functional limit of occupation time fluctuations of branching systems. For example, Li et al [12, 13] studied the branching particle systems with degenerate branching. Miloś [16] investigated the change caused by the varied initial distributions of the classical $(d, \alpha, \beta)$ branching particle systems.

Without other statement, in this paper, we use $M$ to denote an unspecified positive finite constant which may not necessarily be the same in each occurrence, and use $B, B^{H}, \eta^{H}, R^{H}$ to denote the standard Brownian motion, the FBM with index $H$, the Sub-FBM with index $H$ and the RL process with index $H$, respectively.

The remainder of this paper is organized as follows. In Section 2, we report the main results. Section 3 contains some basic calculations. In the last section, i.e. Section 4, we prove the main results.

\section{Main results}

From now on, we always let $N(s)$ and $X_{T}(t)$ be the random counting measure of the $(1, \alpha, \sigma(x))$-particle system and the corresponding scaled occupation time fluctuation process. Let $\xi$ denote the particles' space motion. Then $\xi$ is the symmetric $\alpha$-stable Lévy process. Denote its semigroup by $\left\{L_{t}\right\}_{t \geq 0}$ and the transition density by $p_{t}$, i.e.,

$$
L_{t} f(x):=\mathbb{E}(f(\xi(t+s)) \mid \xi(s)=x)=\int_{\mathbb{R}} p_{t}(y-x) f(y) \mathrm{d} y,
$$

for all $s, t \geq 0, x \in \mathbb{R}$ and bounded measurable functions $f$ (to avoid ambiguity we sometimes write $L_{t} f(x)$ as $\left.L_{t}(f(\cdot))(x)\right)$. For every $\varphi \in \mathcal{S}(\mathbb{R})$, denote the Green potential operator by

$$
G \varphi(x)=\int_{0}^{\infty} L_{s} \varphi(x) \mathrm{d} s
$$

and define

$$
G_{t} \varphi(x)=\int_{0}^{t} L_{s} \varphi(x) \mathrm{d} s
$$

From Li [14,

$$
\mathbb{E}(\langle N(t), \phi\rangle)=\int_{\mathbb{R}} L_{t} \phi(x) \mathrm{d} x=\int_{\mathbb{R}} \phi(x) \mathrm{d} x=\langle\lambda, \phi\rangle .
$$

The occupation time fluctuation process $X_{T}=\left\{X_{T}(t), t \geq 0\right\}$ is rewritten as follows.

$$
\left\langle X_{T}(t), \phi\right\rangle=\frac{1}{F_{T}} \int_{0}^{T t}\langle N(s)-\lambda, \phi\rangle \mathrm{d} s,
$$

for every $\phi \in \mathcal{S}(\mathbb{R})$, where $F_{T}$ is a suitable norming constant.

The main results of this paper read as follows.

Theorem 2.1 Suppose $D:=\int_{\mathbb{R}} \sigma(x) \mathrm{d} x<\infty$ and $\alpha \in(1,2)$. Let $F_{T}^{2}=T^{3-2 / \alpha}$. Then $X_{T} \Rightarrow K \lambda R^{H}$ in $C([0, \tau], \mathbb{R})$ for any $\tau>0$, where $R^{H}(\cdot)$ is a $R L$ process with index $H=3 / 2-1 / \alpha$ and $K=\sqrt{2 \gamma D} \Gamma(1 / \alpha) /[\pi(\alpha-1)]$.

Theorem 2.2 Suppose $D:=\int_{\mathbb{R}} \sigma(x) \mathrm{d} x<\infty$ and $\alpha=1$. Let $F_{T}^{2}=T(\ln T)^{2}$. Then $X_{T} \Rightarrow_{i} C \lambda B$, where $C=2 \sqrt{\gamma D} / \pi$. Here $\Rightarrow_{i}$ means that for each $t>0$, $\int_{0}^{t}\left\langle X_{T}, \psi(\cdot, s)\right\rangle \mathrm{d}$ s converge to $\int_{0}^{t}\langle X, \psi(\cdot, s)\rangle \mathrm{d} s$ in distribution for all $\psi \in \mathcal{S}\left(\mathbb{R}^{2}\right)$. 
The long range dependence of processes can be characterized by means of the so-called dependence exponent which was developed in [6]. According to [6], the dependence exponent $\kappa$ of a Gaussian process $\left\{\zeta_{t} ; t \geq 0\right\}$ is

$$
\kappa=\inf _{0 \leq u<v<s<t} \sup \left\{\gamma>0: \operatorname{Cov}\left(\zeta_{v}-\zeta_{u}, \zeta_{T+t}-\zeta_{T+s}\right)=o\left(T^{-\gamma}\right) \text { as } T \rightarrow \infty\right\} .
$$

The following proposition is of the dependence exponent of RL processes. It seems simple; we write down it because we do not find an appropriate reference.

Proposition 2.1 The dependence exponent $\kappa$ of the $R L$ process $\left\{R^{H}(t), t \geq 0\right\}$ with $H \neq \frac{1}{2}$ is $\frac{3}{2}-H$.

Below we make some comments on our results.

Remark 2.1 (1) From the assumption of $\int_{\mathbb{R}} \sigma(x) \mathrm{d} x<\infty$, one can readily relate the $(1, \alpha, \sigma(x))$-particle systems to the particle systems without branching. Some results of the latter's occupation time fluctuations were revealed in Bojdecki et al [4, 5]. They considered the particle systems where particles start off at time $t=0$ from a Poisson random field with Lebesgue intensity measure $\lambda$ and move independently in $\mathbb{R}^{d}$ with a symmetrical $\alpha$-stable Lévy motion, and proved that if $d / \alpha<1$, then $F_{T}=T^{1-\frac{d}{2 \alpha}}$ and the limit process $X=K \lambda B^{1-\frac{d}{2 \alpha}}$; if $d / \alpha=1$, then $F_{T}=(T \ln T)^{1 / 2}$ and the limit process $X=K \lambda B$. Comparing their results with the corresponding results in this paper, there at least exist two differences in the case of $d=1$. They are, respectively, $F_{T}=T^{1 / 2} \ln T$ vs $F_{T}=(T \ln T)^{1 / 2}$ when $\alpha=1$, and $F_{T}=T^{\frac{3}{2}-\frac{1}{\alpha}}$ plus $R^{\frac{3}{2}-\frac{1}{\alpha}}$ vs $F_{T}=T^{1-\frac{1}{2 \alpha}}$ plus $B^{1-\frac{1}{2 \alpha}}$ when $\alpha \in(1,2)$. The limit processes $R^{\frac{3}{2}-\frac{1}{\alpha}}$ and $B^{1-\frac{1}{2 \alpha}}$ have the same dependence exponent $1 / \alpha$ but different self-similarity indice: $\frac{3}{2}-\frac{1}{\alpha}$ vs $1-\frac{1}{2 \alpha}$, and different increments' property: non-stationary increments vs stationary increments. The difference on the norming $F_{T}$ also shows that the occupation time fluctuations in the $(1, \alpha, \sigma(x))$ model increase faster than those in the particle systems without branching.

(2) It is also interesting to compare the results of $(1, \alpha, \sigma(x))$-particle systems where $\sigma(x) \equiv 1 / 2$ with the results in this paper. In fact, when $\sigma(x) \equiv 1 / 2$, the $(1, \alpha, \sigma(x))$ particle systems are the classical $(d, \alpha, \beta)$-branching particle systems with $d=1, \beta=1$ (see, for example, Bojdecki et al [4]). For the classical $(1, \alpha, 1)$-branching particle systems, Theorem 2.2 in Bojdecki et al [4] (see, also, Bojdecki et al [3] ) told us that a long-range dependent Sub-FBM $\eta^{\frac{3}{2}-\frac{1}{2 \alpha}}$ with dependence exponent $1 / \alpha$ arise from the temporal structure of the limit process of occupation time fluctuations in the case of $\alpha \in(1 / 2,1)$. Moreover, Bojdecki et al intuitively explained the cause of the long range dependence as the "clan recurrence". In this paper, we get the long-range dependent RL process $R^{\frac{3}{2}-\frac{1}{\alpha}}$ in the case of $\alpha \in(1,2)$. The long-range dependence exponent is $1 / \alpha<1$, and the long-range dependence is likely caused by the recurrence of particles' motion.

(3) By the terminology in Bojdecki et al [7], the convergence $\Rightarrow_{i}$ in Theorem 2.2 is called convergence in the space-time, or integral, sense. Bojdecki et al 2 pointed out that $\Rightarrow_{i}$ convergence resembles the convergence of finite-dimensional distributions but neither implies the other. However, by the same proof as in Bojdecki et al [7, we can additionally conclude in Theorem 2.2 that $X_{T}$ converges to $C \lambda B$ in the sense of finite-dimensional distributions. 
At the end of this section, let us show some heuristics to explain why one can expect the RL process arising as a limit in Theorem 2.1. To this end, observe the covariance of $X_{T}$. Let $0 \leq s, t \leq 1$. From (4.2), it is easy to get that

$$
\begin{aligned}
\operatorname{Cov}\left(\left\langle X_{T}(t), \phi\right\rangle,\left\langle X_{T}(s), \phi\right\rangle\right)=\frac{T^{2}}{F_{T}^{2}} \int_{0}^{s} \int_{0}^{t} \operatorname{Cov}(\langle N(T u), \phi\rangle,\langle N(T v), \phi\rangle) \mathrm{d} u \mathrm{~d} v \\
=\frac{2 \gamma T^{3}}{F_{T}^{2}} \int_{0}^{s} \int_{0}^{t} \int_{\mathbb{R}} \sigma(x) \int_{0}^{u \wedge v} L_{T(u-r)} \phi(x) L_{T(v-r)} \phi(x) \mathrm{d} r \mathrm{~d} x \mathrm{~d} u \mathrm{~d} v \\
+\frac{T^{2}}{F_{T}^{2}} \int_{0}^{s} \int_{0}^{t} \int_{\mathbb{R}} \phi(x) L_{T|u-v|} \phi(x) \mathrm{d} x \mathrm{~d} u \mathrm{~d} v .
\end{aligned}
$$

From (3.1) and (3.2) in Bojdecki et al [6], for any $\phi \in \mathcal{S}(\mathbb{R})$, there is constant $M>$ $M_{1}>0$ such that

$$
M_{1} u^{-1 / \alpha} \leq L_{u} \phi(x) \leq M u^{-1 / \alpha}
$$

for any $u>0$. Since $F_{T}^{2}=T^{3-\frac{2}{\alpha}}$ and $1>1 / \alpha$, we have that

$$
\begin{aligned}
\frac{\gamma T^{3}}{F_{T}^{2}} \int_{0}^{s} & \int_{0}^{t} \int_{\mathbb{R}} \sigma(x) \int_{0}^{u \wedge v} L_{T(u-r)} \phi(x) L_{T(v-r)} \phi(x) \mathrm{d} r \mathrm{~d} x \mathrm{~d} u \mathrm{~d} v \\
& \sim \int_{0}^{s} \int_{0}^{t} \mathrm{~d} u \mathrm{~d} v \int_{0}^{u \wedge v}(u-r)^{-1 / \alpha}(v-r)^{-1 / \alpha} \mathrm{d} r \\
& \sim \int_{0}^{s \wedge t}(s-r)^{1-1 / \alpha}(t-r)^{1-1 / \alpha} \mathrm{d} r
\end{aligned}
$$

where $A \sim B$ means that $A / B \in(0, \infty)$, and that

$$
\lim _{T \rightarrow \infty} \frac{T^{2}}{F_{T}^{2}} \int_{0}^{s} \int_{0}^{t} \int_{\mathbb{R}} \phi(x) L_{T|u-v|} \phi(x) \mathrm{d} x \mathrm{~d} u \mathrm{~d} v=0 .
$$

Therefore,

$$
\operatorname{Cov}\left(\left\langle X_{T}(t), \phi\right\rangle,\left\langle X_{T}(s), \phi\right\rangle\right) \sim \int_{0}^{s \wedge t}(s-r)^{1-1 / \alpha}(t-r)^{1-1 / \alpha} \mathrm{d} r
$$

which is the covariance of the RL process $R^{H}$ with $H=3 / 2-1 / \alpha$.

\section{Preliminary calculations}

Define a sequence of random variables $\tilde{X}_{T}$ in $\mathcal{S}^{\prime}\left(\mathbb{R}^{2}\right)$ as follows: For any $T>1$ and $\psi \in \mathcal{S}\left(\mathbb{R}^{2}\right)$, let

$$
\left\langle\tilde{X}_{T}, \psi\right\rangle=\int_{0}^{1}\left\langle X_{T}(t), \psi(\cdot, t)\right\rangle \mathrm{d} t
$$

Without other statement, in the sequel, $\psi \in \mathcal{S}\left(\mathbb{R}^{2}\right)$ always has the form $\psi(x, t)=$ $\phi(x) h(t)$, where $\phi \in \mathcal{S}(\mathbb{R})$ and $h \in \mathcal{S}(\mathbb{R})$ are nonnegative functions. Let

$$
\tilde{h}(s)=\int_{s}^{1} h(t) \mathrm{d} t \text { and } \psi_{T}(x, s)=\frac{1}{F_{T}} \phi(x) \tilde{h}\left(\frac{s}{T}\right) .
$$


Define

$$
V_{\psi_{T}}(x, t, r)=1-\mathbb{E}_{x}\left(\exp \left\{-\int_{0}^{t}\left\langle N(s), \psi_{T}(\cdot, r+s)\right\rangle \mathrm{d} s\right\}\right),
$$

where $\mathbb{E}_{x}(f(N)):=\mathbb{E}\left(f(N) \mid N(0)=\epsilon_{x}\right)$ and $\epsilon_{x}$ denotes the unit measure concentrated at $x \in \mathbb{R}$. From Section 3 in [14, we get that

$$
\begin{aligned}
V_{\psi_{T}}(x, t, r)= & \int_{0}^{t} L_{s}\left[\psi_{T}(\cdot, r+s)\left(1-V_{\psi_{T}}(\cdot, t-s, r+s)\right)\right](x) \mathrm{d} s \\
& -\gamma \int_{0}^{t} L_{s}\left[\sigma(\cdot) V_{\psi_{T}}^{2}(\cdot, t-s, r+s)\right](x) \mathrm{d} s .
\end{aligned}
$$

Furthermore, define

$$
J_{\psi_{T}}(x, t, r):=\int_{0}^{t} L_{s} \psi_{T}(\cdot, r+s)(x) \mathrm{d} s .
$$

Then

$$
V_{\psi_{T}}(x, t, r) \leq J_{\psi_{T}}(x, t, r)
$$

and

$$
\mathbb{E}\left(\mathrm{e}^{-\left\langle\tilde{X}_{T}, \psi\right\rangle}\right)=\exp \left(I_{1}\left(T, \psi_{T}\right)+I_{2}\left(T, \psi_{T}\right)+I_{3}\left(T, \psi_{T}\right)\right),
$$

where

$$
\begin{aligned}
I_{1}\left(T, \psi_{T}\right) & =\gamma \int_{\mathbb{R}} \sigma(x) \mathrm{d} x \int_{0}^{T} J_{\psi_{T}}^{2}(x, T-s, s) \mathrm{d} s \\
I_{2}\left(T, \psi_{T}\right) & =\gamma \int_{\mathbb{R}} \sigma(x) \mathrm{d} x \int_{0}^{T}\left[V_{\psi_{T}}^{2}(x, T-s, s)-J_{\psi_{T}}^{2}(x, T-s, s)\right] \mathrm{d} s \\
& \geq-2 \gamma\left[I_{21}\left(T, \psi_{T}\right)+\gamma I_{22}\left(T, \psi_{T}\right)\right], \\
I_{3}\left(T, \psi_{T}\right) & =\int_{\mathbb{R}} \mathrm{d} x \int_{0}^{T} \psi_{T}(x, s) V_{\psi_{T}}(x, T-s, s) \mathrm{d} s,
\end{aligned}
$$

and

$$
\begin{aligned}
I_{21}\left(T, \psi_{T}\right)= & \int_{\mathbb{R}} \sigma(x) \mathrm{d} x \int_{0}^{T} J_{\psi_{T}}(x, T-s, s) \mathrm{d} s \\
& \times \int_{s}^{T} L_{u-s}\left(\psi_{T}(\cdot, u) J_{\psi_{T}}(\cdot, T-u, u)\right)(x) \mathrm{d} u, \\
I_{22}\left(T, \psi_{T}\right)= & \int_{\mathbb{R}} \sigma(x) \mathrm{d} x \int_{0}^{T} J_{\psi_{T}}(x, T-s, s) \mathrm{d} s \\
& \times \int_{s}^{T} L_{u-s}\left(\sigma(\cdot) J_{\psi_{T}}^{2}(\cdot, T-u, u)\right)(x) \mathrm{d} u .
\end{aligned}
$$

3.1 Limits of $I_{1}, I_{2}$ and $I_{3}$ when $1<\alpha<2$ 
Step 1. We consider the limit of $I_{1}\left(T, \psi_{T}\right)$. From (3.8) and (3.5), we get that

$$
\begin{aligned}
I_{1}\left(T, \psi_{T}\right) & =\gamma \int_{\mathbb{R}} \sigma(x) \mathrm{d} x \int_{0}^{T}\left(\int_{0}^{T-s} L_{u} \psi_{T}(x, s+u) \mathrm{d} u\right)^{2} \mathrm{~d} s \\
& =\int_{0}^{1} h(t) \mathrm{d} t \int_{0}^{1} h(r) \mathrm{d} r \int_{0}^{r \wedge t} \Psi_{T}(s, r, t) \mathrm{d} s
\end{aligned}
$$

where

$$
\Psi_{T}(s, r, t)=\frac{T \gamma}{F_{T}^{2}} \int_{\mathbb{R}} \sigma(x) \mathrm{d} x \int_{0}^{T(t-s)} L_{u} \phi(x) \mathrm{d} u \int_{0}^{T(r-s)} L_{v} \phi(x) \mathrm{d} v .
$$

The self-similarity of the $\alpha$-stable Lévy process $\xi$ implies that

$$
\frac{1}{T^{1-\frac{1}{\alpha}}} \int_{0}^{T t} L_{u} f(x) \mathrm{d} u=\int_{0}^{t} \int_{\mathbb{R}} u^{-1 / \alpha} p_{1}\left((x-y) T^{-1 / \alpha} u^{-1 / \alpha}\right) f(y) \mathrm{d} y,
$$

for any integrable function $f$. This means that for every $0<t \leq 1$ and non-negative integrable function $f$

$$
\frac{\alpha}{\alpha-1} t^{1-\frac{1}{\alpha}} p_{1}(0) \int_{\mathbb{R}} f(y) \mathrm{d} y \geq \frac{1}{T^{1-\frac{1}{\alpha}}} \int_{0}^{T t} L_{u} f(x) \mathrm{d} x .
$$

Additionally, as $T \rightarrow \infty$,

$$
\frac{1}{T^{1-\frac{1}{\alpha}}} \int_{0}^{T t} L_{u} f(x) \mathrm{d} x \rightarrow \frac{\alpha}{\alpha-1} t^{1-\frac{1}{\alpha}} p_{1}(0) \int_{\mathbb{R}} f(y) \mathrm{d} y .
$$

Therefore, (3.14) and the facts that $\int_{\mathbb{R}} \sigma(x) \mathrm{d} x<\infty$ and $F_{T}^{2}=T^{3-2 / \alpha}$ lead to

$$
\begin{aligned}
\Psi_{T}(s, r, t) & \rightarrow \gamma\left(p_{1}(0) \frac{\alpha}{\alpha-1}\right)^{2}[(t-s)(r-s)]^{1-1 / \alpha} \int_{\mathbb{R}} \sigma(x) \mathrm{d} x\left(\int_{\mathbb{R}} \phi(x) \mathrm{d} x\right)^{2} \\
& =C[(t-s)(r-s)]^{1-1 / \alpha}\left(\int_{\mathbb{R}} \phi(x) \mathrm{d} x\right)^{2}
\end{aligned}
$$

as $T \rightarrow \infty$, where

$$
C=\gamma\left(\frac{\alpha p_{1}(0)}{\alpha-1}\right)^{2} \int_{\mathbb{R}} \sigma(x) \mathrm{d} x=\gamma\left(\frac{\Gamma(1 / \alpha)}{\pi(\alpha-1)}\right)^{2} \int_{\mathbb{R}} \sigma(x) \mathrm{d} x .
$$

Consequently, from (3.13) we can readily have that as $T \rightarrow \infty$,

$$
I_{1}\left(T, \psi_{T}\right) \rightarrow C\left(\int_{\mathbb{R}} \phi(x) \mathrm{d} x\right)^{2} \int_{0}^{1} h(t) \mathrm{d} t \int_{0}^{1} h(r) \mathrm{d} r \int_{0}^{r \wedge t}[(t-s)(r-s)]^{1-1 / \alpha} \mathrm{d} s .
$$

Step 2. We consider the limit of $I_{2}\left(T, \psi_{T}\right)$. Using (3.2), (3.5) and the fact that $\tilde{h}$ is bounded, we have that

$$
J_{\psi_{T}}(x, T-u, u) \leq \frac{M}{F_{T}} G_{T} \phi(x)
$$


for any $u \leq T$ and some $M>0$. Applying this inequality to (3.11) and using (3.2) again, we get a constant $M>0$ such that

$$
\begin{aligned}
I_{21}\left(T, \psi_{T}\right) & \leq \frac{M T}{F_{T}^{3}} \int_{\mathbb{R}} \sigma(x) \int_{0}^{T} L_{v} \phi(x) \mathrm{d} v \int_{0}^{T} L_{u}\left(\phi \int_{0}^{T} L_{w} \phi \mathrm{d} w\right)(x) \mathrm{d} u \mathrm{~d} x \\
& \leq \frac{M T}{F_{T}^{3}} \int_{\mathbb{R}} \sigma(x) G_{T} \phi(x) G_{T}\left(\phi G_{T} \phi\right)(x) \mathrm{d} x .
\end{aligned}
$$

It follows from (3.15) that for any non-negative integrable function $f$ there exists a constant $M>0$ such that

$$
G_{T} f(x)<M T^{1-1 / \alpha} .
$$

Therefore, substituting $F_{T}^{2}=T^{3-2 / \alpha}$ into (3.18), we can readily obtain that

$$
I_{21}\left(T, \psi_{T}\right) \leq \frac{M}{T^{1 / 2}} \int_{\mathbb{R}} \sigma(x) \mathrm{d} x \rightarrow 0 .
$$

Furthermore, applying (3.17) and (3.2) to (3.12), we obtain that

$$
I_{22}\left(T, \psi_{T}\right) \leq \frac{M T}{F_{T}^{3}} \int_{\mathbb{R}} \sigma(x)\left(G_{T} \phi(x)\right)^{2} G_{T}\left(\sigma G_{T} \phi\right)(x) \mathrm{d} x,
$$

for some $M>0$. From (3.19), (3.21) and the fact $\int_{\mathbb{R}} \sigma(x) \mathrm{d} x<\infty$, it follows that

$$
I_{22}\left(T, \psi_{T}\right) \leq \frac{M}{T^{1 / \alpha-1 / 2}} \rightarrow 0,
$$

where the convergence is due to the fact $\alpha \in(1,2)$. Note that $I_{2}\left(T, \psi_{T}\right) \leq 0$ which follows from (3.6). Combining (3.9) with (3.18) and (3.22) yields that as $T \rightarrow \infty$,

$$
I_{2}\left(T, \psi_{T}\right) \rightarrow 0 .
$$

Step 3. At last we pass to the limit of $I_{3}\left(T, \psi_{T}\right)$. Let

$$
I_{31}\left(T, \psi_{T}\right):=\int_{\mathbb{R}} \mathrm{d} x \int_{0}^{T} \psi_{T}(x, s) J_{\psi_{T}}(x, T-s, s) \mathrm{d} s .
$$

From (3.10), (3.6), (3.17) and (3.19), it follows that

$$
\begin{aligned}
0 \leq I_{3}\left(T, \psi_{T}\right) & \leq I_{31}\left(T, \psi_{T}\right) \leq \frac{M T}{F_{T}^{2}} \int_{\mathbb{R}} \phi(x) G_{T} \phi(x) \mathrm{d} x \\
& \leq \frac{T^{2-1 / \alpha} M}{F_{T}^{2}} \int_{\mathbb{R}} \phi(x) \mathrm{d} x,
\end{aligned}
$$

for some $M>0$. Substituting $F_{T}^{2}=T^{3-2 / \alpha}$ into (3.25), $\alpha \in(1,2)$ indicates that

$$
I_{3}\left(T, \psi_{T}\right) \rightarrow 0
$$

3.2 Limits of $I_{1}, I_{2}$ and $I_{3}$ when $\alpha=1$ 
We first point out that

$$
\lim _{T \rightarrow \infty} I_{2}\left(T, \psi_{T}\right)=0
$$

and

$$
\lim _{T \rightarrow \infty} I_{3}\left(T, \psi_{T}\right)=0 .
$$

The details are similar to those of $I_{2}$ and $I_{3}$ in Section 3.1, and hence we omit them. Below, we discuss $I_{1}\left(T, \psi_{T}\right)$.

From (3.47) in [8] we know that for any $0<t \leq 1$,

$$
\lim _{T \rightarrow \infty} \frac{1}{\ln T} \int_{0}^{T t} L_{u} \phi(x) \mathrm{d} u=p_{1}(0) \int_{\mathbb{R}} \phi(x) \mathrm{d} x .
$$

Using this fact and noting that $\int_{\mathbb{R}} \sigma(x) \mathrm{d} x<\infty$ and $F_{T}^{2}=T(\ln T)^{2}$, we get that

$$
\lim _{T \rightarrow \infty} \Psi_{T}(s, r, t)=\gamma\left[p_{1}(0) \int_{\mathbb{R}} \phi(x) \mathrm{d} x\right]^{2} \int_{\mathbb{R}} \sigma(x) \mathrm{d} x,
$$

where $\Psi_{T}(s, r, t)$ is the same as (3.14). Note that (3.19) and the integrability of $\sigma(x)$ imply the boundedness of $\Psi_{T}(s, r, t)$ with respect to $T>0,0 \leq r, t \leq 1$ and $0 \leq s \leq r \wedge t$. Therefore, the dominated convergence theorem and (3.13) imply that as $T \rightarrow \infty$,

$$
I_{1}\left(T, \psi_{T}\right) \rightarrow C_{1}\left(\int_{\mathbb{R}} \phi(x) \mathrm{d} x\right)^{2} \int_{0}^{1} h(t) \mathrm{d} t \int_{0}^{1} h(r)(r \wedge t) \mathrm{d} r
$$

where

$$
C_{1}=\gamma\left(p_{1}(0)\right)^{2} \int_{\mathbb{R}} \sigma(x) \mathrm{d} x=\frac{\gamma}{\pi^{2}} \int_{\mathbb{R}} \sigma(x) \mathrm{d} x
$$

\section{Proofs of the main results}

Proof of Theorem 2.1 Without loss of generality, fix $\tau=1$. As explained in Bojdecki et al 4, to prove this theorem it is sufficient to show that

(i) For each non-negative $\psi \in \mathcal{S}\left(\mathbb{R}^{d+1}\right)$, as $T \rightarrow \infty$

$$
\mathbb{E}\left(\mathrm{e}^{-\left\langle\tilde{X}_{T}, \psi\right\rangle}\right) \rightarrow \exp \left(\frac{1}{2} \int_{0}^{1} \int_{0}^{1} \operatorname{Cov}(\langle X(s), \psi(\cdot, s)\rangle,\langle X(t), \psi(\cdot, t)\rangle) \mathrm{d} s \mathrm{~d} t\right),
$$

where $\tilde{X}_{n}$ and $\tilde{X}$ are defined as (3.1) and $X$ is the corresponding limit process;

(ii) $\left\{\left\langle X_{T}, \phi\right\rangle ; T \geq 1\right\}$ is tight in $C([0,1], \mathbb{R})$ for any given $\phi \in \mathcal{S}(\mathbb{R})$, where the theorem of Mitoma [17] is used.

If $\psi(x, t)=\phi(x) h(t)$, where $\phi \in \mathcal{S}(\mathbb{R})$ and $h \in \mathcal{S}(\mathbb{R})$ are nonnegative functions, then the limit process in Theorem 2.1 satisfies that

$$
\begin{gathered}
\operatorname{Cov}(\langle X(s), \psi(\cdot, s)\rangle,\langle X(t), \psi(\cdot, t)\rangle)=K^{2}\langle\lambda, \phi\rangle^{2} h(s) h(t) \mathbb{E}(R(s) R(t)) \\
=K^{2}\langle\lambda, \phi\rangle^{2} h(s) h(t) \int_{0}^{s \wedge t}[(t-u)(s-u)]^{1-1 / \alpha} \mathrm{d} u
\end{gathered}
$$


where $K^{2}=2 \gamma \int_{\mathbb{R}} \sigma(x) \mathrm{d} x\left(\frac{\Gamma(1 / \alpha)}{\pi(\alpha-1)}\right)^{2}$. Furthermore, combining (3.7) with (3.23), (3.16) and $(3.26)$ yields

$$
\begin{aligned}
& \lim _{T \rightarrow \infty} \mathbb{E}\left(\mathrm{e}^{-\left\langle\tilde{X}_{T}, \psi\right\rangle}\right) \\
& =\exp \left(\gamma\left(\frac{\Gamma\left(\frac{1}{\alpha}\right)\langle\lambda, \phi\rangle}{\pi(\alpha-1)}\right)^{2} \int_{\mathbb{R}} \sigma(x) \mathrm{d} x \int_{0}^{1} \int_{0}^{1} h(s) h(t) \int_{0}^{s \wedge t}[(t-u)(s-u)]^{1-\frac{1}{\alpha}} \mathrm{d} u \mathrm{~d} s \mathrm{~d} t\right) \\
& =\exp \left(\frac{1}{2} \int_{0}^{1} \int_{0}^{1} \operatorname{Cov}(\langle X(s), \psi(\cdot, s)\rangle,\langle X(t), \psi(\cdot, t)\rangle) \mathrm{d} s \mathrm{~d} t\right) .
\end{aligned}
$$

This means that (4.1) holds for the special case $\psi(x, t)=\phi(x) h(t)$.

For general $\psi$, the proof of (i) is the same with slightly more complicated notation. The details are omitted.

We now pass to prove (ii). Note that by some simple and standard arguments, one can readily get that for any $f, g \in \mathcal{S}(\mathbb{R})$ and $s \leq t$,

$$
\mathbb{E}_{x}(\langle N(s), f\rangle\langle N(t), g\rangle)=L_{s}\left(f L_{t-s} g\right)(x)+2 \gamma \int_{0}^{s} L_{u}\left(\sigma L_{s-u} f L_{t-u} g\right)(x) \mathrm{d} u .
$$

Thus, by Poisson initial condition,

$\operatorname{Cov}(\langle N(s), f\rangle,\langle N(t), g\rangle)=\int_{\mathbb{R}} f(x) L_{t-s} g(x) \mathrm{d} x+2 \gamma \int_{\mathbb{R}} \sigma(x) \int_{0}^{s} L_{s-u} f(x) L_{t-u} g(x) \mathrm{d} u \mathrm{~d} x$.

From the setting of $X_{T}(t)$, we have that for any $0 \leq s<t \leq 1$,

$$
\begin{aligned}
& \mathbb{E}\left(\left\langle X_{T}(t), \phi\right\rangle-\left\langle X_{T}(s), \phi\right\rangle\right)^{2}=\frac{T^{2}}{F_{T}^{2}} \int_{s}^{t} \int_{s}^{t} \operatorname{Cov}(\langle N(T u), \phi\rangle,\langle N(T v), \phi\rangle) \mathrm{d} u \mathrm{~d} v \\
&= \frac{2 \gamma T^{3}}{F_{T}^{2}} \int_{s}^{t} \int_{s}^{t} \int_{\mathbb{R}} \sigma(x) \int_{0}^{u \wedge v} L_{T(u-r)} \phi(x) L_{T(v-r)} \phi(x) \mathrm{d} r \mathrm{~d} x \mathrm{~d} u \mathrm{~d} v \\
&+\frac{T^{2}}{F_{T}^{2}} \int_{s}^{t} \int_{s}^{t} \int_{\mathbb{R}} \phi(x) L_{T|u-v|} \phi(x) \mathrm{d} x \mathrm{~d} u \mathrm{~d} v .
\end{aligned}
$$

Applying the last inequality of (2.4) to the last two formulas of (4.3), noting that $F_{T}^{2}=T^{3-\frac{2}{\alpha}}$ and $\sigma(x)$ is integrable, we get that for all $T>1$

$$
\begin{aligned}
& \frac{2 \gamma T^{3}}{F_{T}^{2}} \int_{s}^{t} \int_{s}^{t} \int_{\mathbb{R}} \sigma(x) \int_{0}^{u \wedge v} L_{T(u-r)} \phi(x) L_{T(v-r)} \phi(x) \mathrm{d} r \mathrm{~d} x \mathrm{~d} u \mathrm{~d} v \\
& \quad \leq M \int_{s}^{t} \mathrm{~d} u \int_{s}^{t} \mathrm{~d} v \int_{0}^{u \wedge v}(u-r)^{-1 / \alpha}(v-r)^{-1 / \alpha} \mathrm{d} r \\
& \quad \leq M \int_{s}^{t} \mathrm{~d} u \int_{s}^{t}|u-v|^{-1 / \alpha} \mathrm{d} v \\
& \quad=M(t-s)^{2-\frac{1}{\alpha}} \int_{0}^{1} \int_{0}^{1}|u-v|^{-1 / \alpha} \mathrm{d} u \mathrm{~d} v \leq M(t-s)^{2-\frac{1}{\alpha}},
\end{aligned}
$$

and

$$
\begin{aligned}
& \frac{T^{2}}{F_{T}^{2}} \int_{s}^{t} \int_{s}^{t} \int_{\mathbb{R}} \phi(x) L_{T|u-v|} \phi(x) \mathrm{d} x \mathrm{~d} u \mathrm{~d} v \\
& \quad \leq \frac{M T^{2-\frac{1}{\alpha}}}{F_{T}^{2}} \int_{s}^{t} \int_{s}^{t}|u-v|^{-1 / \alpha} \mathrm{d} u \mathrm{~d} v \leq M(t-s)^{2-\frac{1}{\alpha}}
\end{aligned}
$$


hold for constants $M>0$ which only depend on $\phi$.

Combining (4.3) with (4.4) and (4.5), we get that

$$
\mathbb{E}\left(\left\langle X_{T}(t), \phi\right\rangle-\left\langle X_{T}(s), \phi\right\rangle\right)^{2} \leq M(\phi)(t-s)^{2-\frac{1}{\alpha}} .
$$

Therefore, from Billingsley [1, Theorem 12.3] and the fact $\left\langle X_{T}(0), \phi\right\rangle=0$, it follows that $\left\{\left\langle X_{T}, \phi\right\rangle\right\}_{T \geq 1}$ is tight in $C([0,1], \mathbb{R})$. The proof of Theorem 2.1 is complete.

Proof of Theorem 2.2. The proof of Theorem 2.2 is easy. In fact, combining (3.7) with (3.27), (3.28) and (3.31) we can readily get the corresponding formula (4.1). More details are omitted.

At last, we give the proof of Proposition 2.1.

Proof of Proposition 2.1. From the representation (1.1) of $R^{H}(\cdot)$, it is easy to see that for any $0 \leq u<v<s<t$,

$$
\begin{aligned}
& \operatorname{Cov}(R(v)-R(u), R(T+t)-R(T+s)) \\
& =\int_{0}^{\infty}\left[(v-r)^{H-\frac{1}{2}} \mathbf{1}_{\{r \leq v\}}-(u-r)^{H-\frac{1}{2}} \mathbf{1}_{\{r \leq u\}}\right]\left[(T+t-r)^{H-\frac{1}{2}}-(T+s-r)^{H-\frac{1}{2}}\right] \mathrm{d} r .
\end{aligned}
$$

By Taylor expansion formula, it is easy to see that for any given $t, s, r$,

$$
\Psi(T, t, s, r):=\frac{\left[(T+t-r)^{H-\frac{1}{2}}-(T+s-r)^{H-\frac{1}{2}}\right]}{T^{H-\frac{3}{2}}} \rightarrow\left(H-\frac{1}{2}\right)(t-s),
$$

as $T \rightarrow \infty$. Consequently, one can readily have that

$$
\begin{aligned}
& \lim _{T \rightarrow \infty} \frac{\operatorname{Cov}(R(v)-R(u), R(T+t)-R(T+s))}{T^{H-\frac{3}{2}}} \\
& =\left(H-\frac{1}{2}\right)(t-s) \int_{0}^{\infty}\left[(v-r)^{H-\frac{1}{2}} \mathbf{1}_{\{r \leq v\}}-(u-r)^{H-\frac{1}{2}} \mathbf{1}_{\{r \leq u\}}\right] \mathrm{d} r \\
& =\frac{2 H-1}{2 H+1}\left(v^{H+\frac{1}{2}}-u^{H+\frac{1}{2}}\right)(t-s),
\end{aligned}
$$

which and (2.3) together imply that the dependence exponent of RL process $R^{H}(\cdot)$ with $H \neq \frac{1}{2}$ is $\frac{3}{2}-H$.

\section{Acknowledgements}

The author thanks the anonymous referees for careful reading of the paper and for the useful suggestions which have helped to improve the paper significantly.

\section{References}

[1] Billingsley, P. Convergence of Probability Measures, Wiley, New York, 1968.

[2] Bojdecki, T., Gorostiza, L, and Ramaswami, S. Convergence of $\mathcal{S}^{\prime}$-valued processes and space-time random fields. J. Funct. Anal., 66 (1986): 21-41.

[3] Bojdecki, T., Gorostiza, L, and Talarczyk, A. Sub-fractional Brownian motion and its relation to occupation times. Statist. Probab. Lett., 69 (2004): 405-419. 
[4] Bojdecki, T., Gorostiza, L, and Talarczyk, A. Limit theorem for occupation time fluctuations of branching systems I: Long-range dependence. Stochastic Process. Appl., 116 (2006): 1-18.

[5] Bojdecki, T., Gorostiza, L, and Talarczyk, A. Limit theorem for occupation time fluctuations of branching systems II: Critical and large dimensions. Stochastic Process. Appl., 116 (2006): 19-35.

[6] Bojdecki, T., Gorostiza, L, and Talarczyk, A. A long range dependence stable process and an infinite variance branching system. Ann. Probab., 35 (2007): 500-527.

[7] Bojdecki, T., Gorostiza, L, and Talarczyk, A. Occupation time fluctuations of an infinitevariance branching system in large dimension. Bernoulli, 13 (2007): 20-39.

[8] Bojdecki, T., Gorostiza, L, and Talarczyk, A. Occupation time limits of inhomogeneous Poisson systems of independent particles. Stochastic Process. Appl., 118 (2008): 28-52.

[9] Dzhaparidze, K.O. and van Zanten, J.H. A series expansion of fractional Brownian motion. Probab. Theory Related Fields 130 (2004): 39-55.

[10] Helga, S. An optimal wavelet series expansion of the Riemann-Liouville process. J. Theoret. Probab., 22 (2009): 1030-1057.

[11] Lévy, P. Random functions: General theory with special reference to Laplacian random functions. Univ. California Publ. Statist., 1(1953) 331-390

[12] Li, Y. and Xiao, Y. Occupation time fluctuations of weakly degenerate branching systems. J. Theoret. Probab. DOI: 10.1007/s10959-011-0358-3

[13] Li, Y. Occupation time fluctuations of strongly degenerate branching systems. Sci. China Ser. A Mathematics. 54 (2011): 2669-2682.

[14] Li, Y. Fluctuation limits of site-dependent Branching systems in critical and large dimensions. Stat. Probab. Lett., 81 (2011): 1604-1611.

[15] Lifshits, M. Linde, W. and Shi, Z. Small deviations of Riemann-Liouville processes in $L_{q}$-space with respect to fractal measures. Proc. London Math. Soc., 92 (2006): 224-250.

[16] Miloś, P. Occupation time fluctuations of Poisson and equilibrium finite variance branching systems. Probab. Math. Statist., 27 (2007): 181-203.

[17] Mitoma, I. Tightness of probability on $C\left([0,1] ; \mathscr{S}^{\prime}\right)$ and $D\left([0,1] ; \mathscr{S}^{\prime}\right)$. Ann. Probab. 11 (1983), 989-999.

[18] Sithi, V.M. and Lim, S.C. On the spectra of Riemann-Liouville fractional Brownian motion. J. phys. A Math. Gen., 28 (1995): 2995-3003. 